\title{
Estimation of Hemodynamic Response Function in the Brain and Brain Tumors: Comparison of Inverse Logistic and Canonical Hemodynamic Response Function Models
}

Seyedeh Mahboobe Seyed Abbasi', Seyed Salman Zakariaee ${ }^{2}$, Abbas Rahimiforoushani ${ }^{*}$

${ }^{1}$ Department of Epidemiology and Biostatistics, Faculty of Public Health, Tehran University of Medical Sciences, Tehran, Iran ${ }^{2}$ Department of Medical Physics, Faculty of Medicine, Kurdistan University of Medical Sciences, Sanandaj, Iran

\section{A BSTRACT}

Introduction: The hemodynamic response function (HRF), reflecting cerebral blood flow in response to neural activity, plays a crucial role in the analysis of the brain data obtained by functional magnetic resonance imaging (fMRI). In this study, a comparison of two statis tical models was performed to evaluate HRF for block design. Materials and Methods: fMRI data from 3 patients with brain tumor were taken using a 3 Tesla scanner. Analysis of fMRI data was performed by the SPM12 toolbox in MATLAB software. The AIC, SBC and MSE indices were used to select the most convenient HRF mode. Results: Based on the simulation data, HRF estimated by canonical HRF model plus time derivations (TD) model was more consistent with simulated HRF. These models were evaluated on real data. The MSE, AIC and SBC indices were obtained for TD-logistic model (IL) models (for TD and logistic IL models; 0.052, 1235.1, 1223.9 and 0.068, -1091.5, 1049.2, respectively). Based on the average values of T, W, H and model selection indicators, IL model for estimating HRF in healthy regions of the brain and brain tumor is a more appropriate approach. Conclusion: The results of the present study can be helpful for the evaluation and diagnosis of HRF in high-metabolism points. Using the IL model to estimate HRF in the block design may lead to a better estimation of HRF and thus maintaining patient health and quality of life after surgical treatment and non-surgical medical procedures.

\section{Key words:}

1. Magnetic Resonance Imaging

2. Logistic Models

3. Brain

*Corresponding Author: Abbas Rahimiforoushani

E-mail: rahimifo@tums.ac.ir 


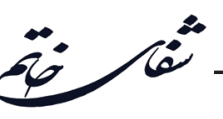

\title{
بر آورد تابع پاسخ هموديناميك در مغز و تومورهاى مغزى: مقايسءٔ مدلهاى لجستيك معكوس و تابع

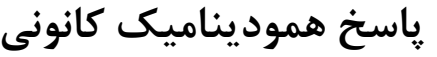

\author{
سيده محبوبه سيدعباسى'، سيد سلمان ذكريايى'، عباس رحيمى فروشانى" \\ اكروه إيبدميولوزى و آمار زيستى، دانشكده بهداشت، دانشكاه علوم يزشكى تهران، تهران، ايران \\ "َّروه فيزيك يزشكى، دانشكده يزشكى، دانشكاه علوم يزشكى كردستان، سنندج، ايران
}

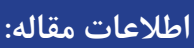

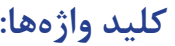

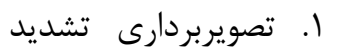
مغناطيسى

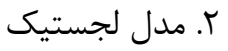

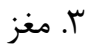

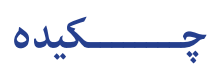

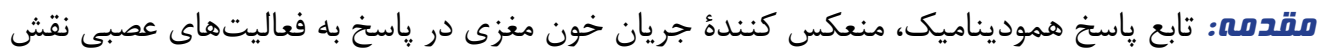

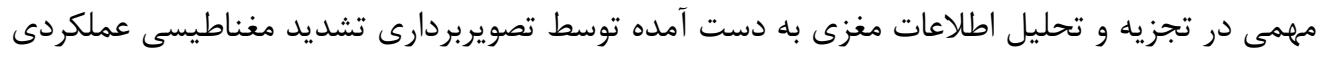

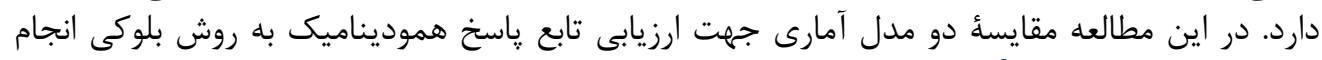

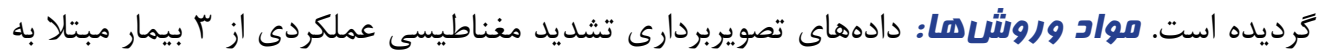

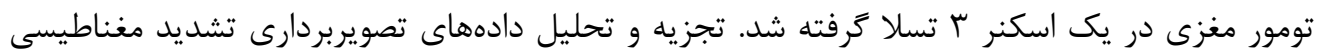

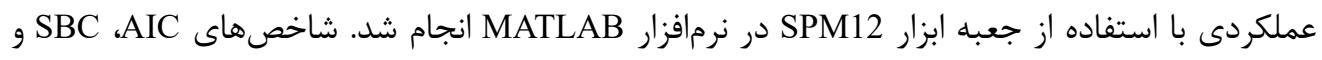

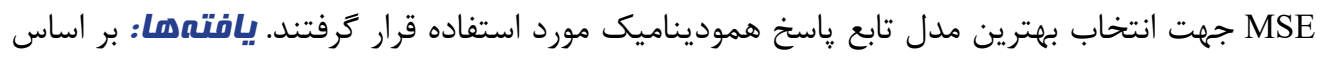

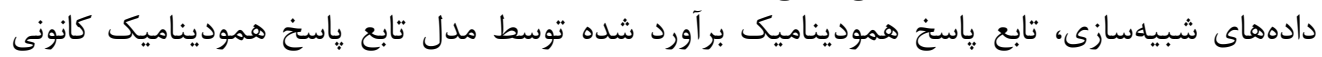

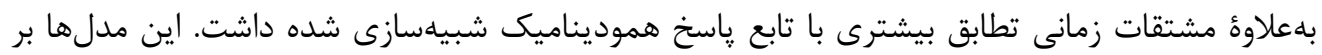

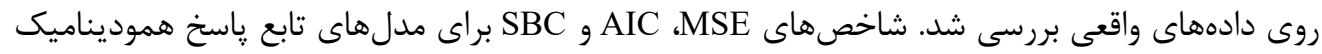

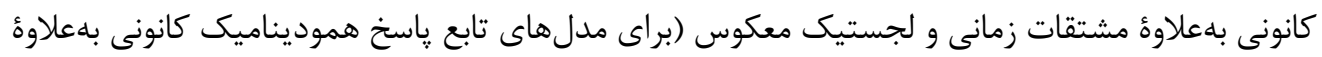

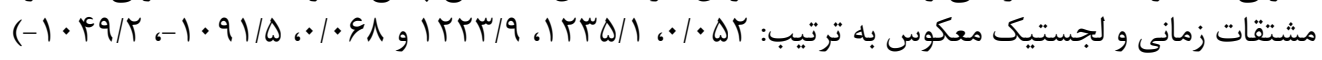

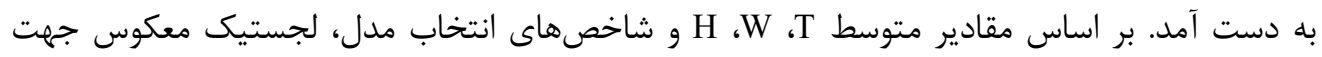

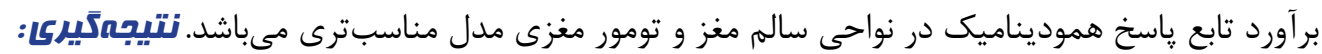

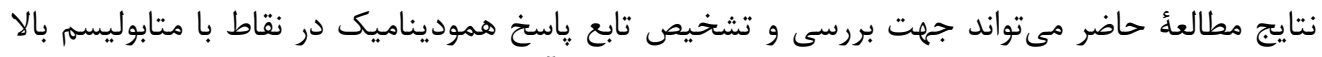

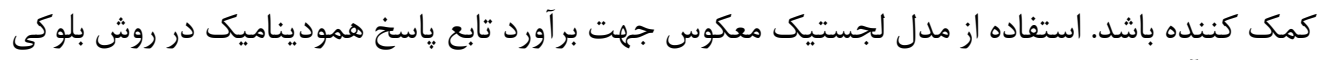

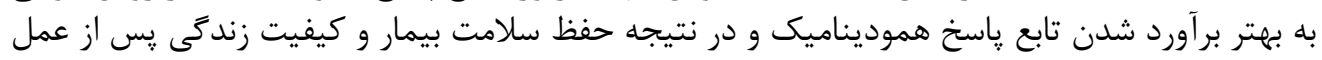

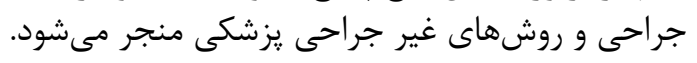

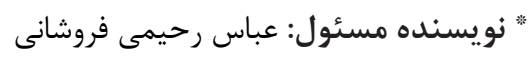
آدرس الكترونيكى: rahimifo@tums.ac.ir 


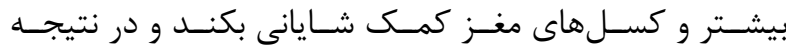

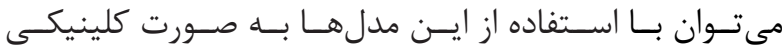

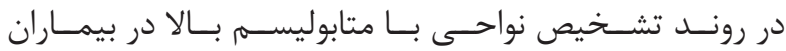

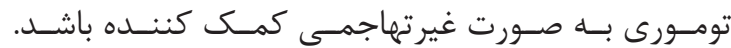

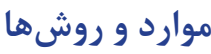

مدل هاى HRF

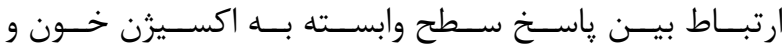

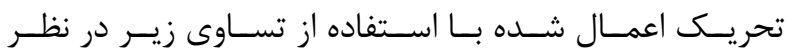

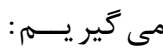

$$
Y=(s * h)(t)
$$

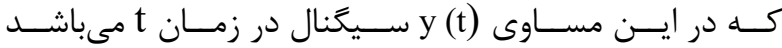

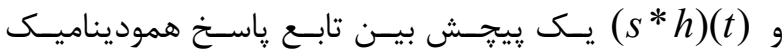

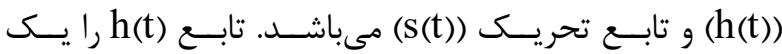

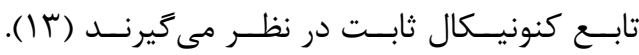

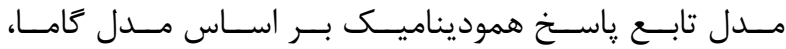

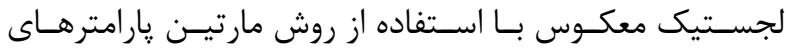

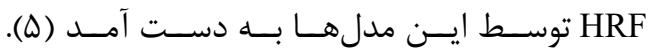

$$
\text { معيار هاى انتخاب مدل }
$$

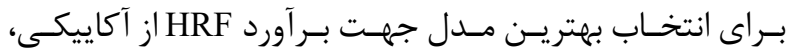

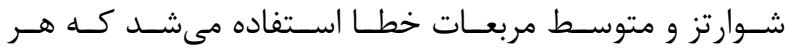

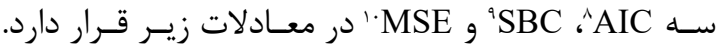

$$
\begin{aligned}
A I C & =n \ln (S S E)-n \ln n+2 p \\
S B C & =n \ln (S S E)-n \ln n+2(\ln n) \\
M S E & =\sum_{i=1}^{n} e_{i}^{2} /(n-2)
\end{aligned}
$$

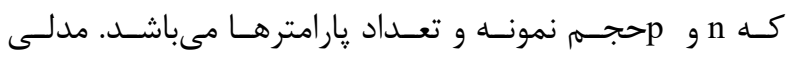

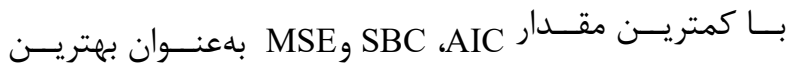

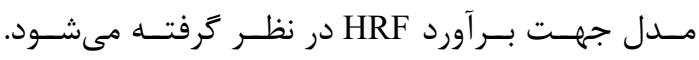
شبيهسازى دادههاى fMIRI

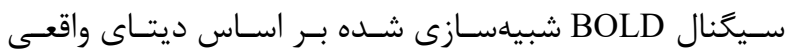

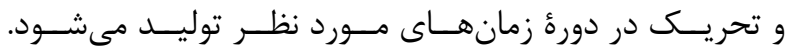

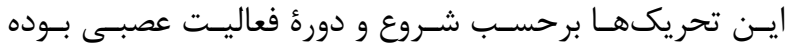

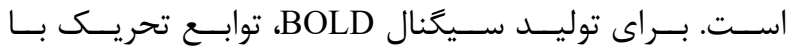

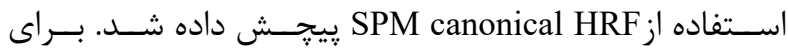

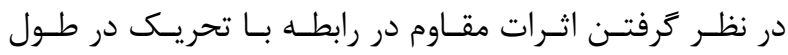

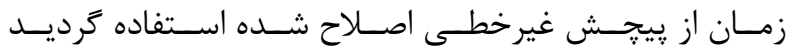

${ }^{1}$ Function magnetic resonance imaging

${ }^{2}$ Blood oxygen level dependent

${ }^{3}$ Hemodynamic response imaging

${ }^{4}$ Cerebral blood flow

${ }^{5}$ Time to peak

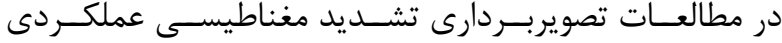

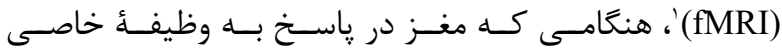

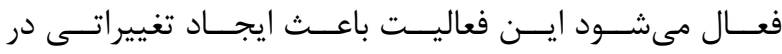

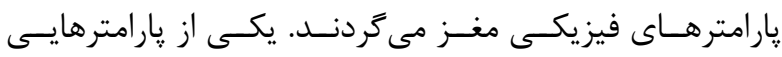

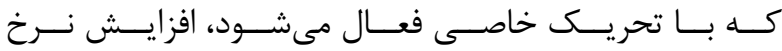

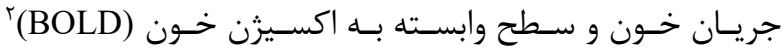

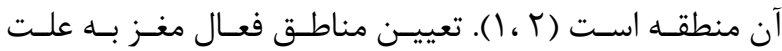

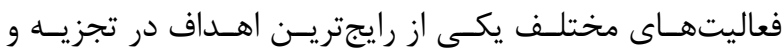

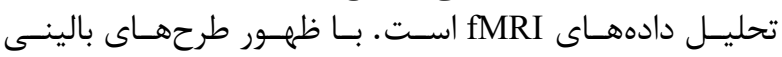

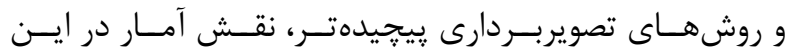

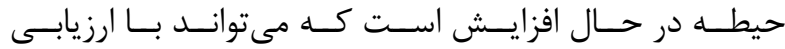

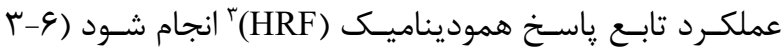

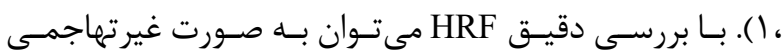

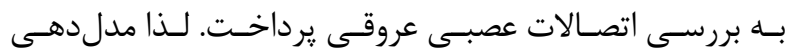

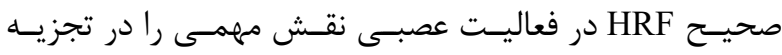

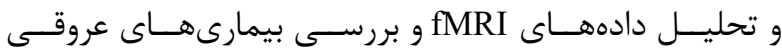

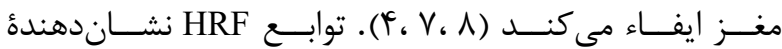

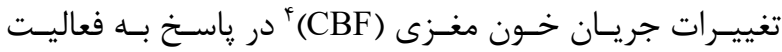

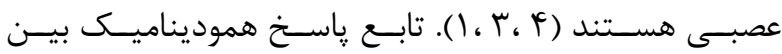

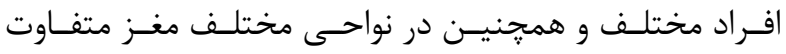

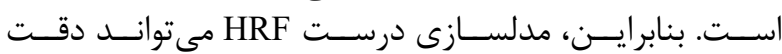

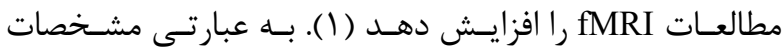

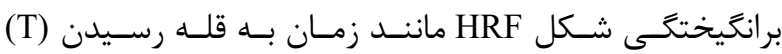

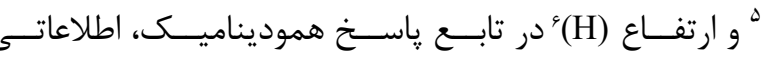

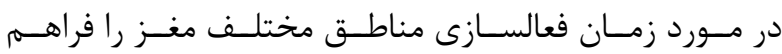

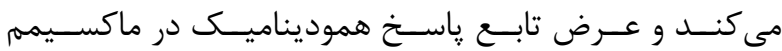

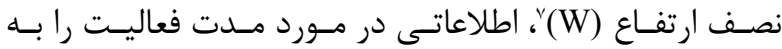

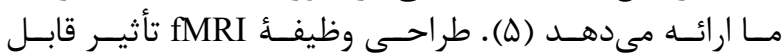

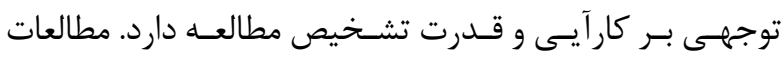

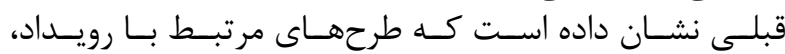

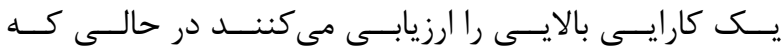

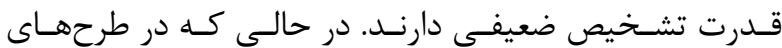

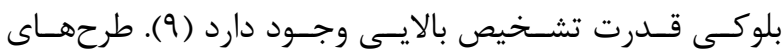

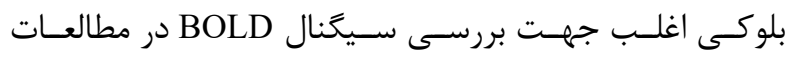

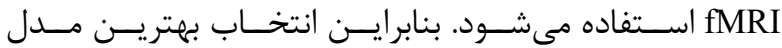

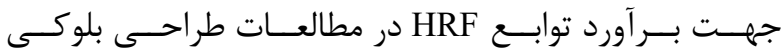

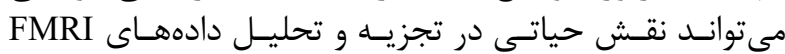

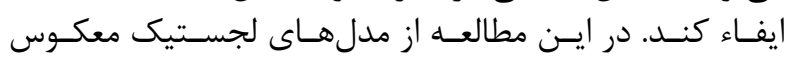

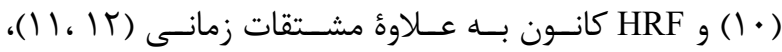

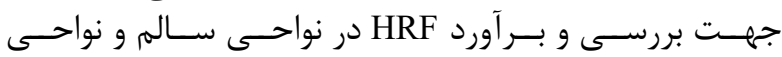

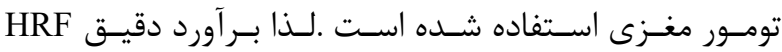

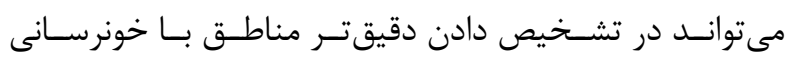

${ }^{6}$ Height

${ }^{7}$ Full width at half maximum

${ }^{8}$ Akaike's information criterion

${ }^{9}$ Schwarz' Bayesian

${ }^{10}$ Mean square error 
(Math works, ver.8.6)

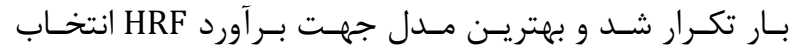

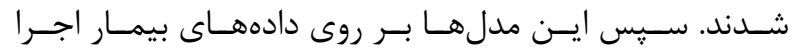

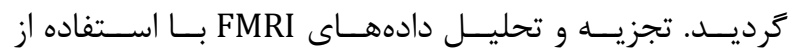

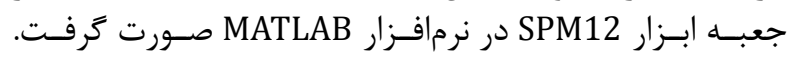

دادهاى بيمار

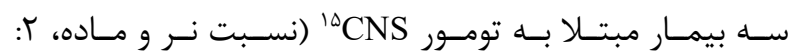

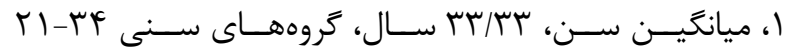

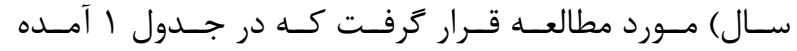

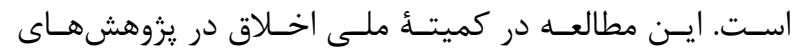

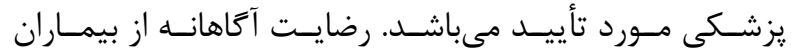

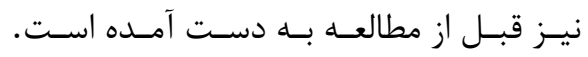
جدول ا- مربوط به اطلاعات بالينى و هيستوياتولوريك بيماران.

\begin{tabular}{|c|c|c|c|}
\hline جنس & سن & نوع تومور & بيمار \\
\hline زن & rr & كَليوما & 1 \\
\hline مرد & rF & كليوما & $r$ \\
\hline مرد & rI & آسترووسايتوما & $r$ \\
\hline
\end{tabular}

يافتهها

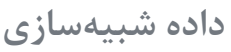

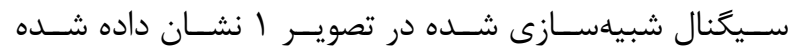

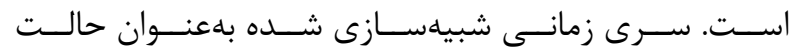

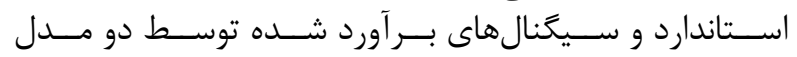

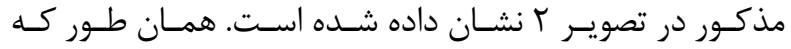

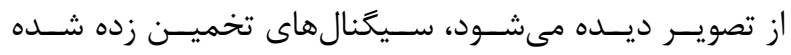

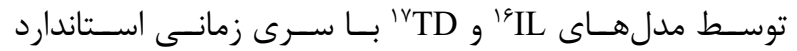
تطابـق خوبــى دارنـد.

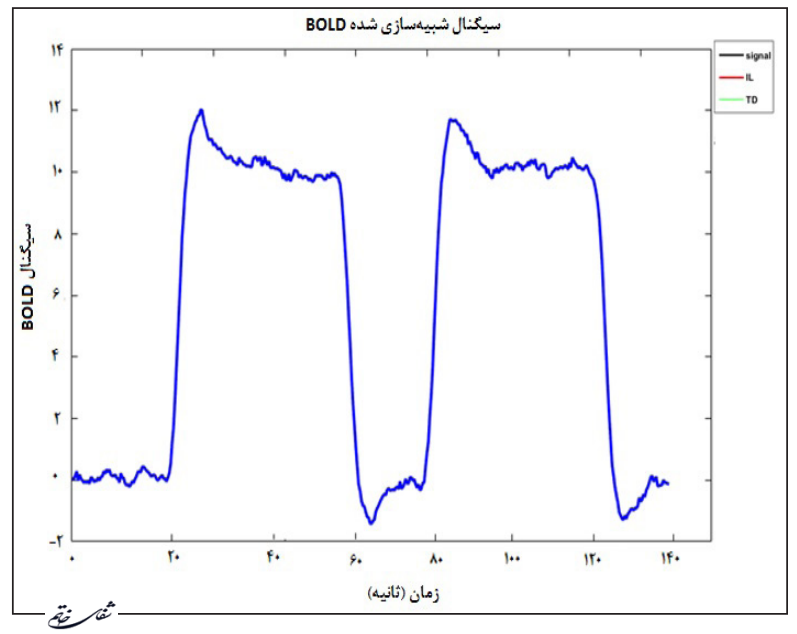

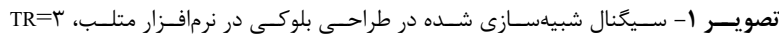

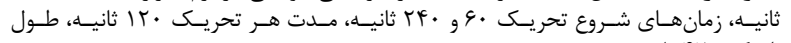

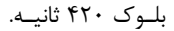

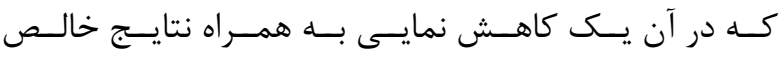

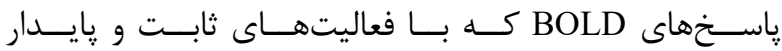

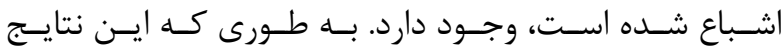

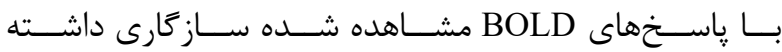

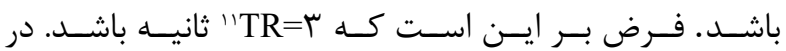

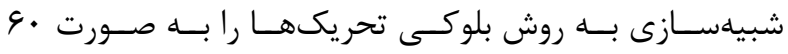

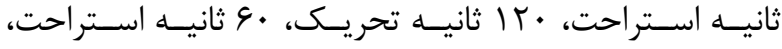

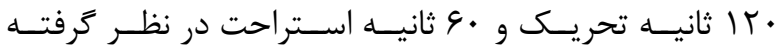

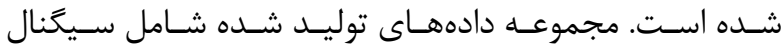

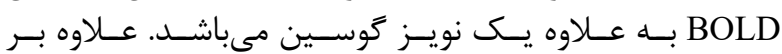

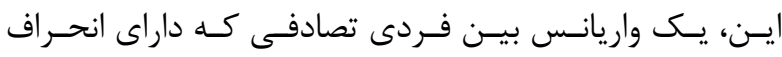

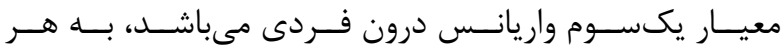

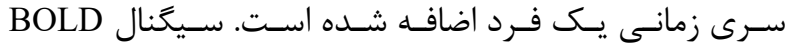

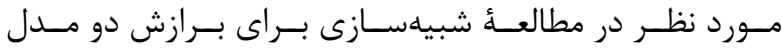

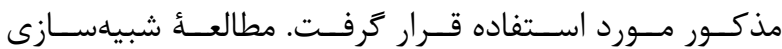

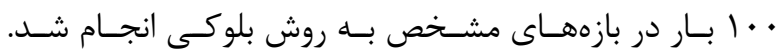

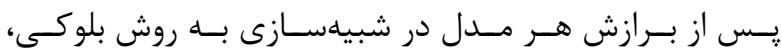

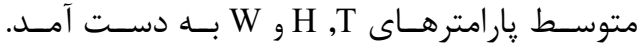

Sاده هاى اكتساب شده fMRI

دادههـاى fMRI در اسـكنر ب تسـلا زيمنـس (مونيـخ، آلمـان)

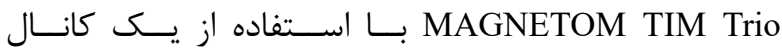

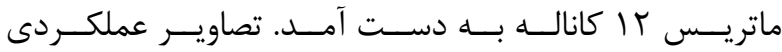

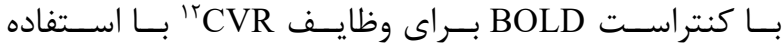

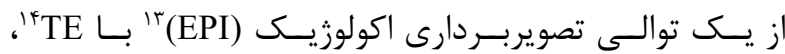

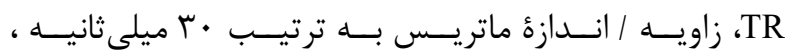

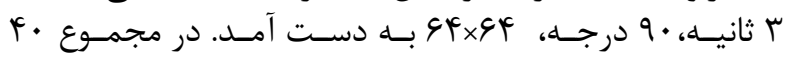

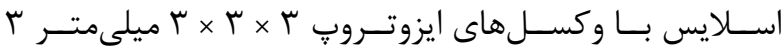

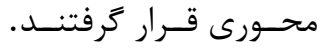

flMRI طراحى وظيفه در تصويربردارى

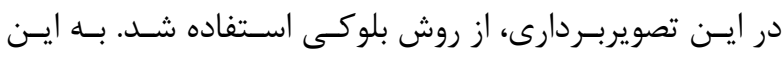

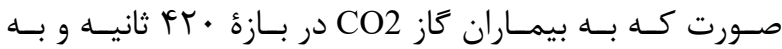

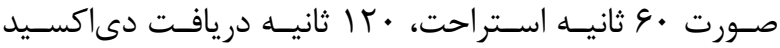

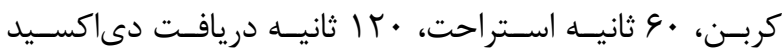

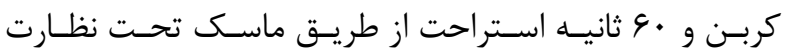

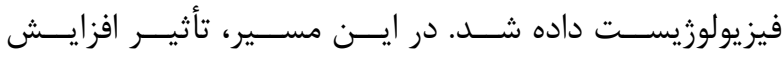

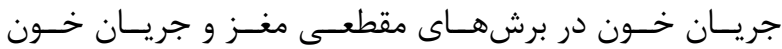

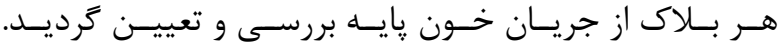

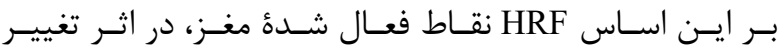

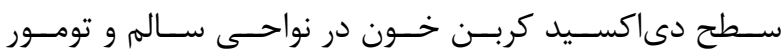
تعييـن كرديـد.

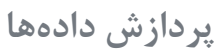

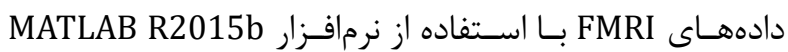

\footnotetext{
${ }^{15}$ Central nervous system tumor

${ }^{16}$ Inverse logistic

${ }^{17}$ Canonical HRF model plus its time derivations
} 


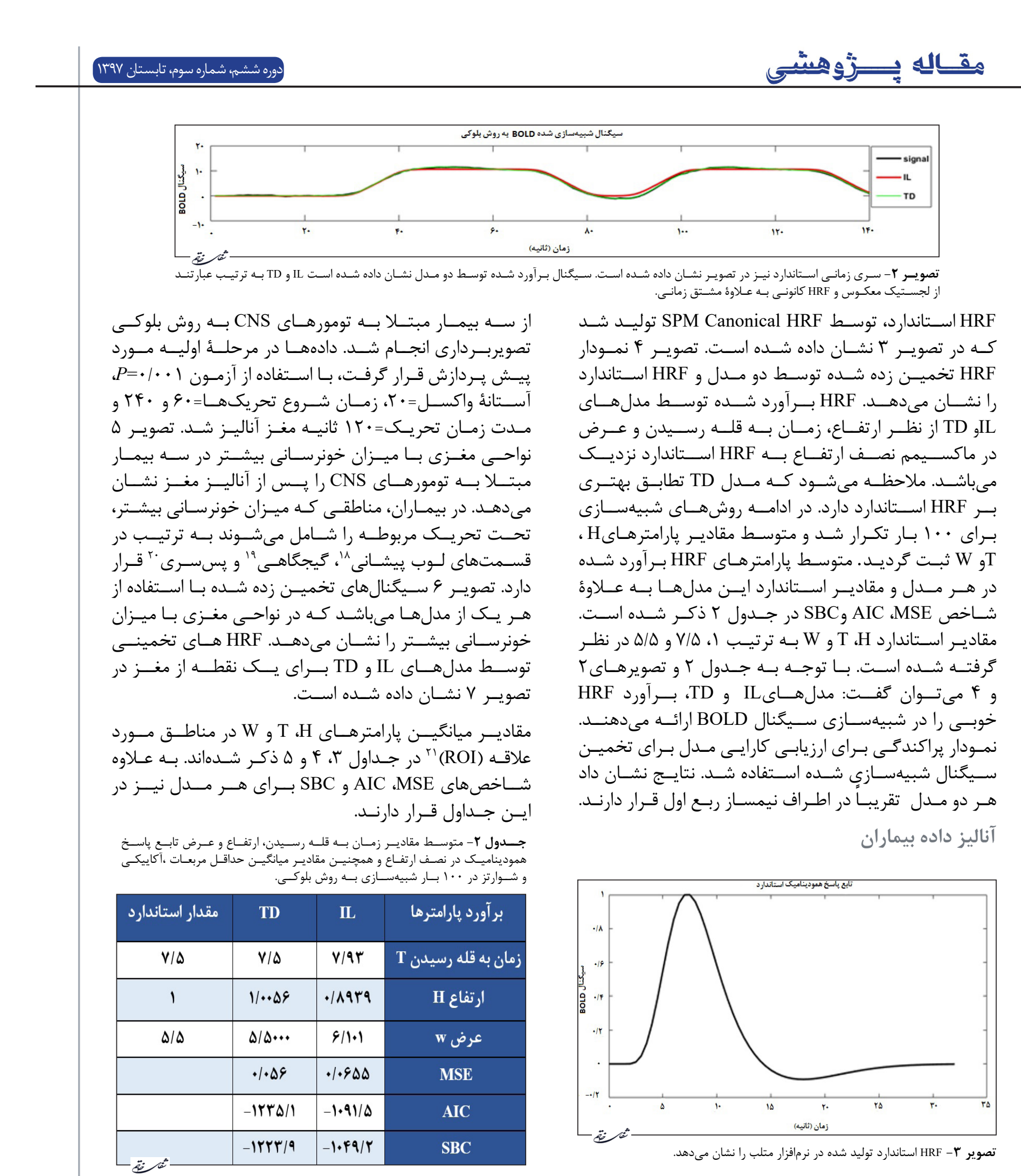

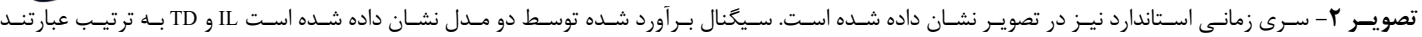

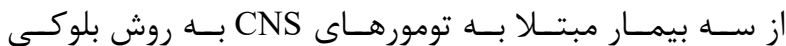

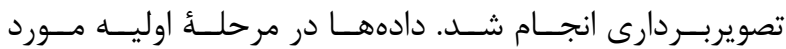

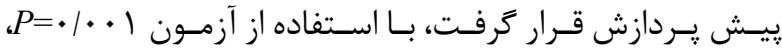

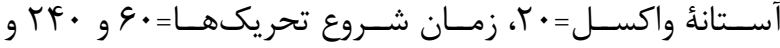

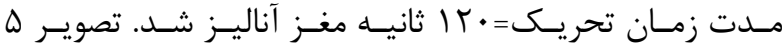

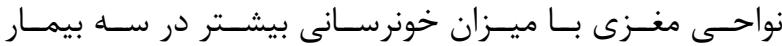

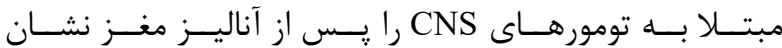

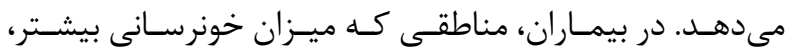

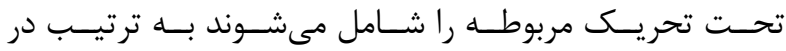

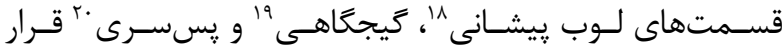

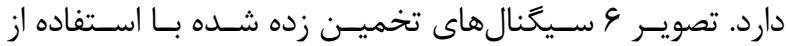

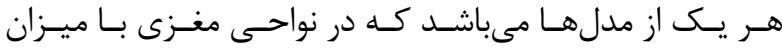

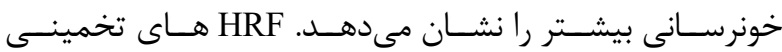

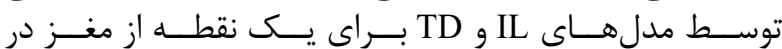

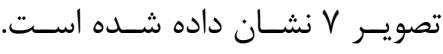

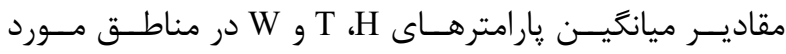

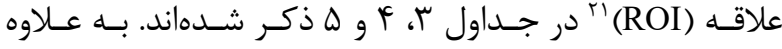

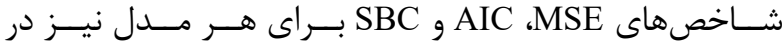

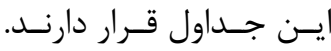

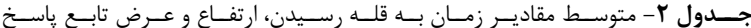

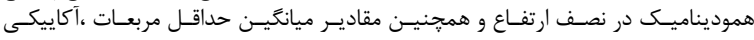

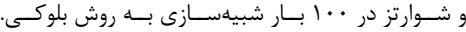

\begin{tabular}{|c|c|c|c|}
\hline مقدار استاندارد & TD & IL & بر آورد هارامترها \\
\hline$V / Q$ & $V / D$ & $v / 9 r$ & زمان به قله رسيدن T \\
\hline 1 & $1 / \cdot+\Delta q$ & - /1 9 rq & ارتفاع H \\
\hline \multirow[t]{4}{*}{$\Delta / \Delta$} & $\Delta / \Delta+\cdots$ & $9 / 1+1$ & عرض w w \\
\hline & $\cdot 1 \cdot \Delta 9$ & $.1 .9 \Delta \Delta$ & MSE \\
\hline & $-1 r r \Delta / 1$ & $-1.91 / 0$ & AIC \\
\hline & -Irry/9 & $-1+f q / r$ & SBC \\
\hline
\end{tabular}

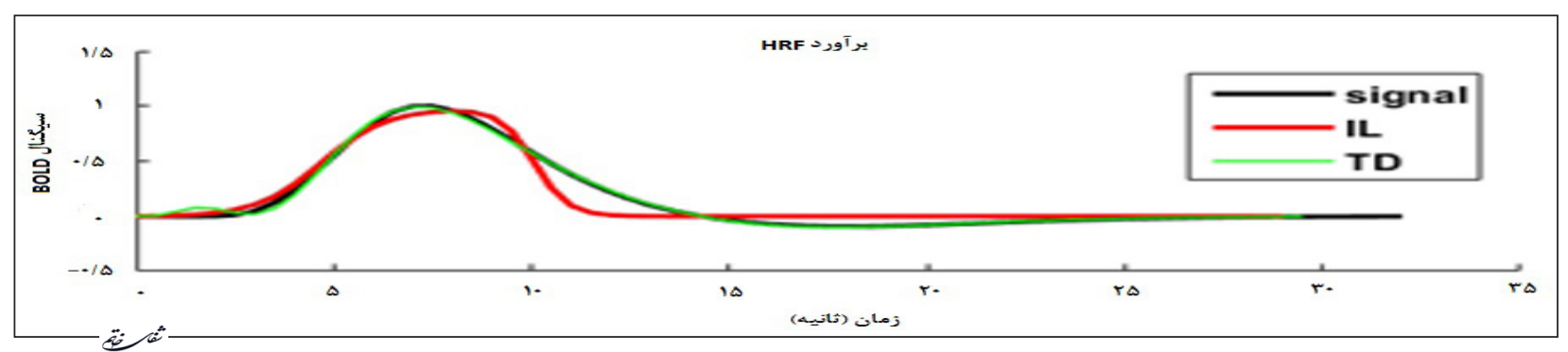

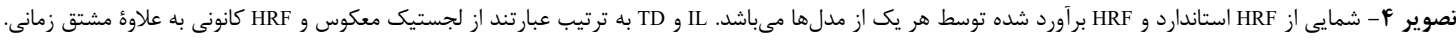

${ }^{18}$ Frontal lobe

${ }^{19}$ Temporal

${ }^{20}$ Occipital

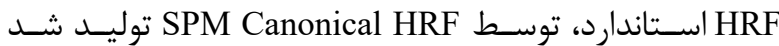

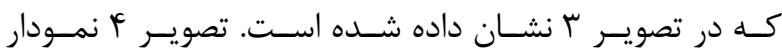

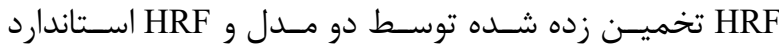

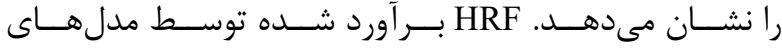

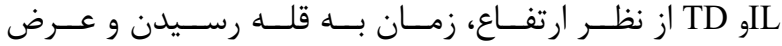

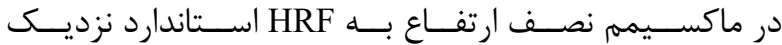

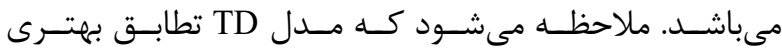

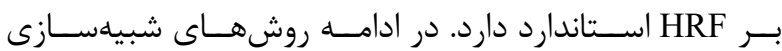

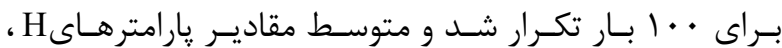

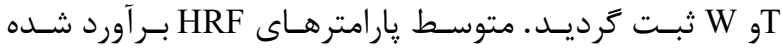

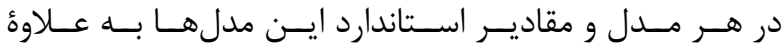

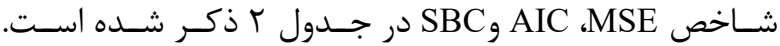

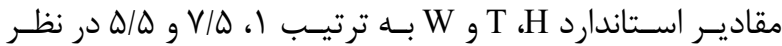

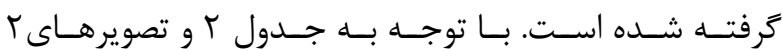

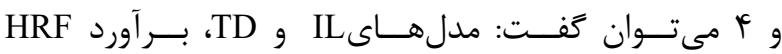

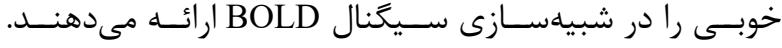

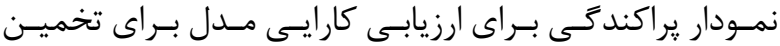

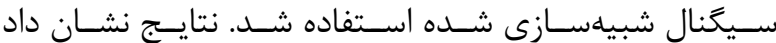

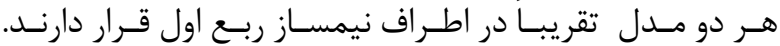

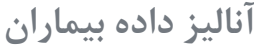

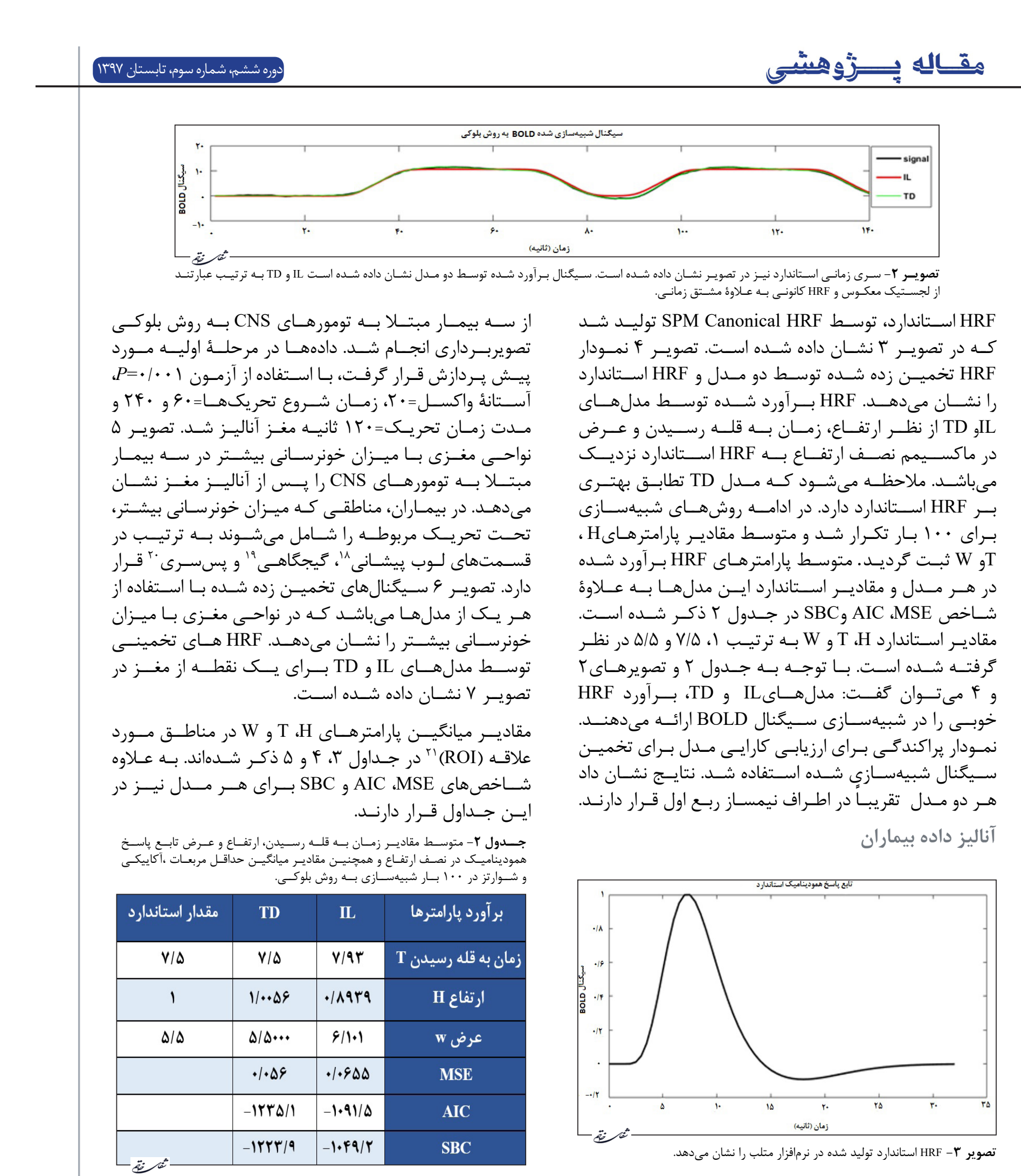

كانونى به علاوه مستى زمانى 

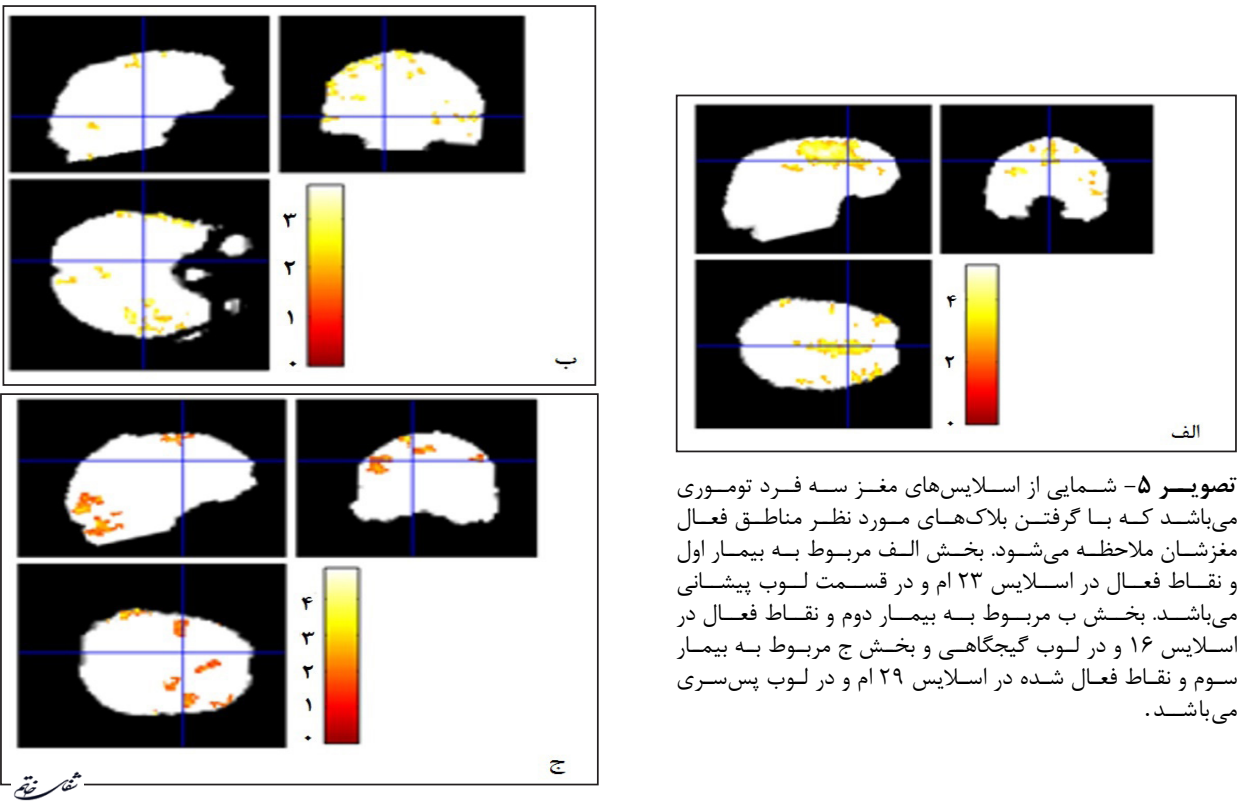

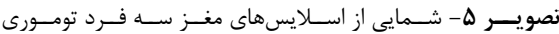

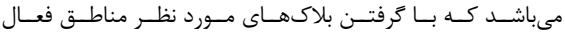

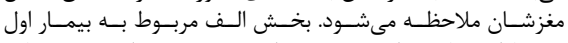

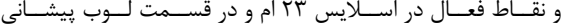

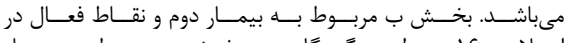

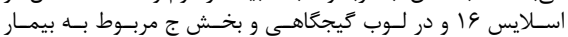

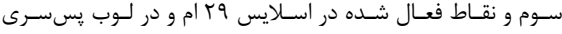
مى باشــد.

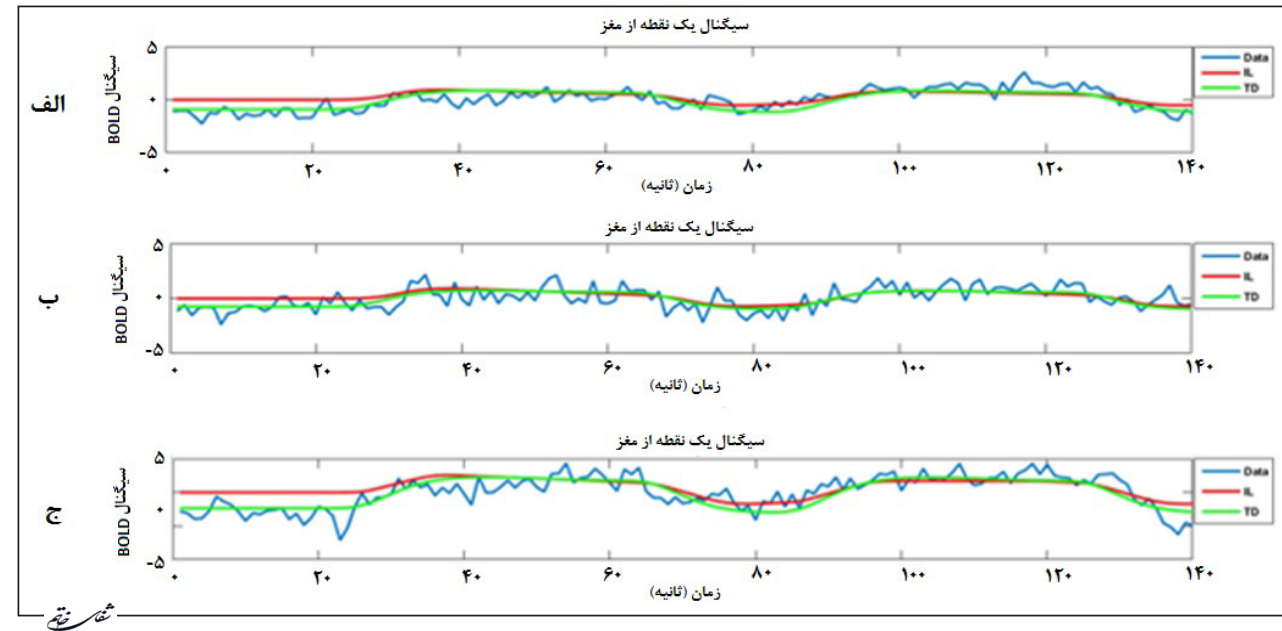

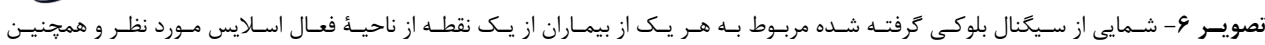

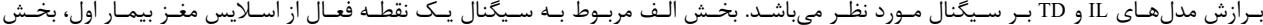

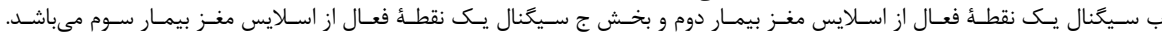

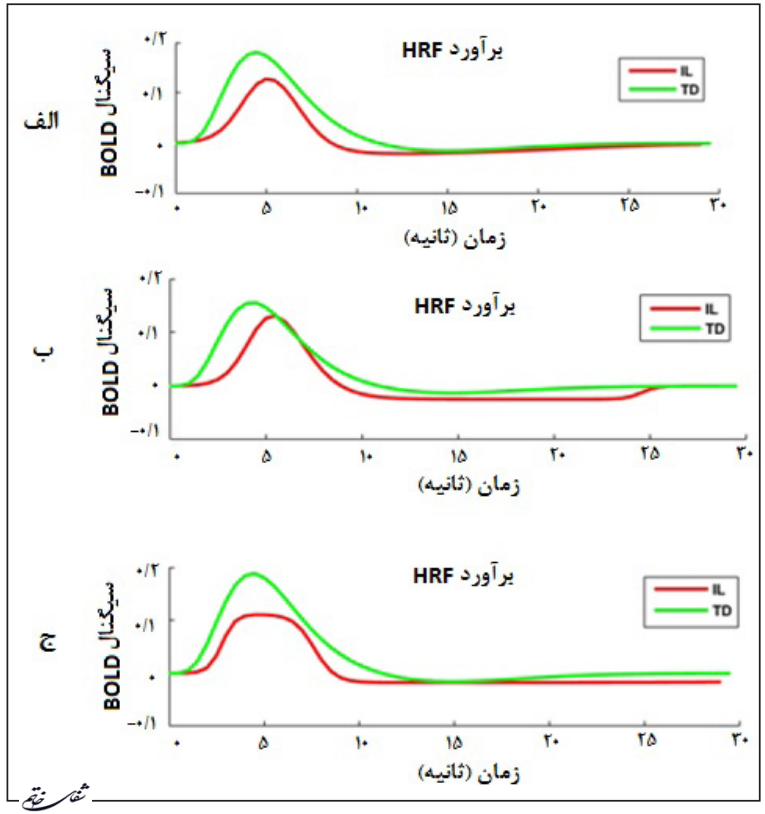

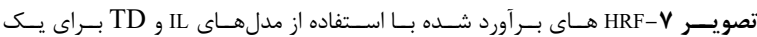

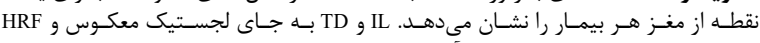

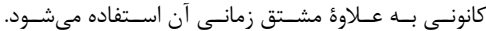


بحث و نتيجه كَيرى

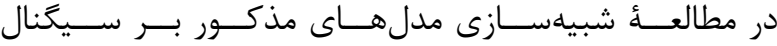

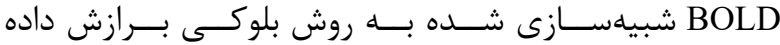

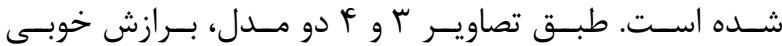

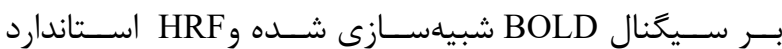

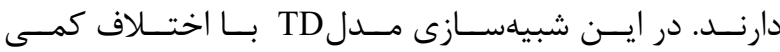

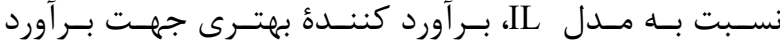

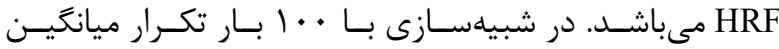

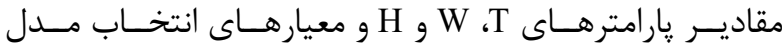

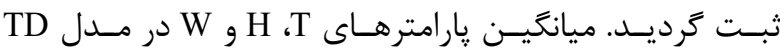

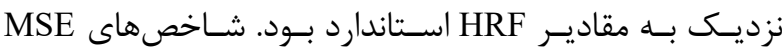
SBC و AIC

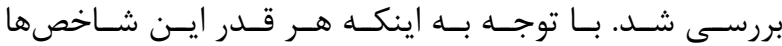

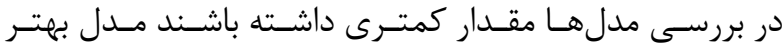

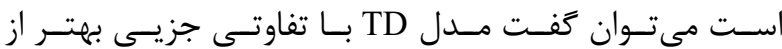

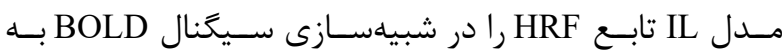

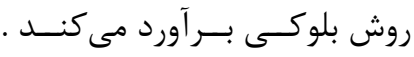

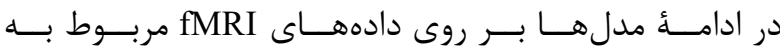

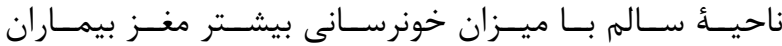

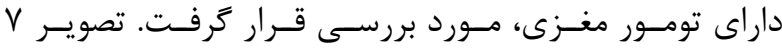

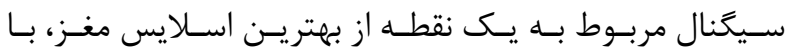

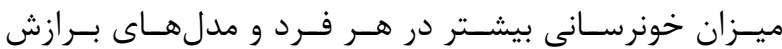

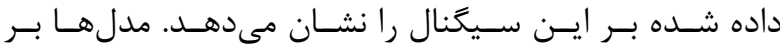

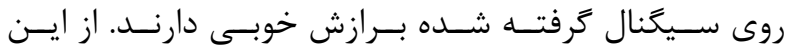

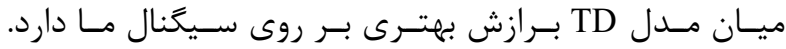

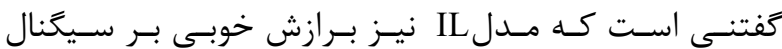

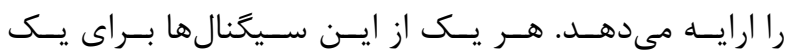

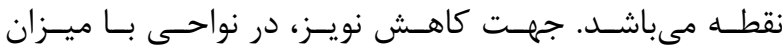

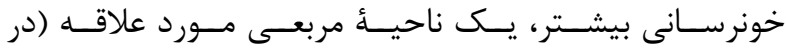

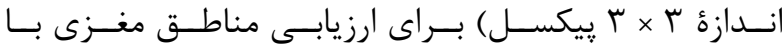

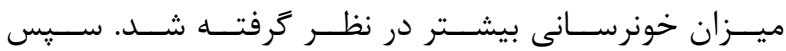

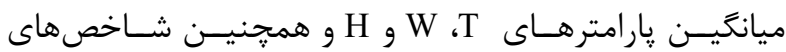

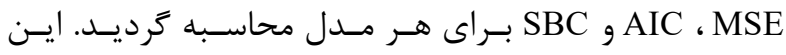

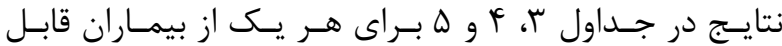

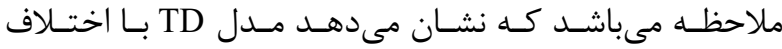

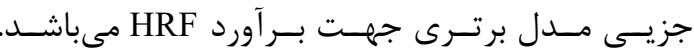

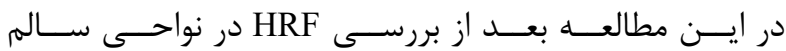

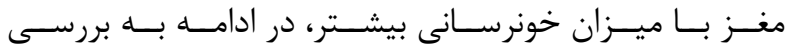

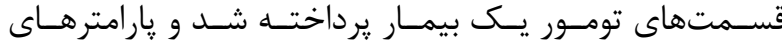

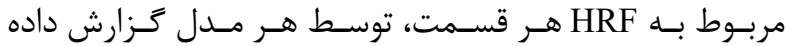

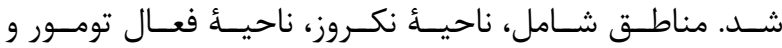

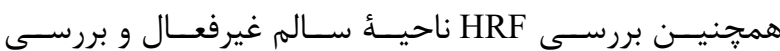

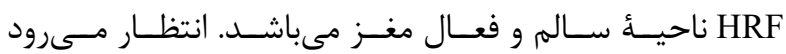

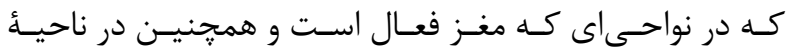

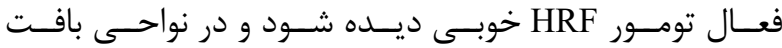

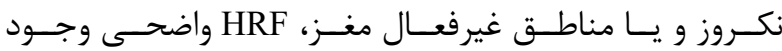

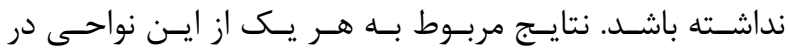

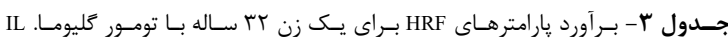

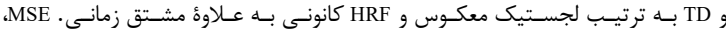

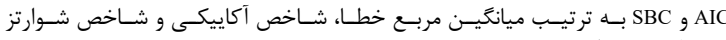

\begin{tabular}{|c|c|c|}
\hline مدل TD & مدل IL & برآورد متوسط بار امترها \\
\hline.$/ 1 Y 1914$ & $\cdot / \cdot V A \Delta V V$ & ار ارتفاع \\
\hline$r / / 9 \Delta \& \Delta T$ & F/AFrrA & زمان به قله رسيدن \\
\hline r/. Frequ & r/queVAr & عرض ع ع \\
\hline 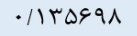 & - / frret & MSE \\
\hline -rVT/ANAN & 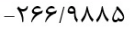 & AIC \\
\hline$-r \varphi \Delta / .9 \mu \wedge$ & 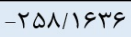 & SBC \\
\hline
\end{tabular}

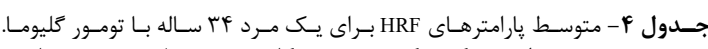

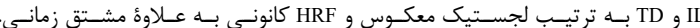

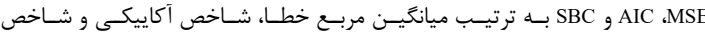

\begin{tabular}{|c|c|c|}
\hline مدل TD & مصل IL & برآورد متوسط ؛ارامترها \\
\hline ./1rq4aा &.$/ 191 \& 4$ & 1رتفاع \\
\hline F/TSOTYT & $F / r, \ldots 1$ & زمان به قله رسيدن \\
\hline$r / 1 r r \Delta q \Lambda$ & T/TYYYA & عرض \\
\hline ./.人ßar & .1 .91 .99 & MSE \\
\hline -MTF/GYAG & $-r r \cdot|f| g r$ & $\mathbf{A I C}$ \\
\hline - YTS/AIYV & $-r r / / \Delta q / F$ & SBC \\
\hline
\end{tabular}

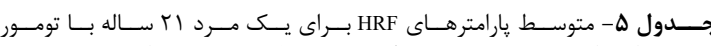

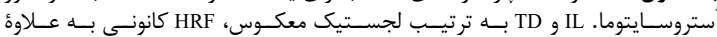

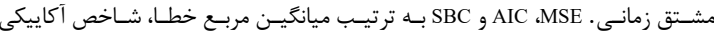

\begin{tabular}{|c|c|c|}
\hline مدل TD & مل IL & بر آورد متوسط بار امتر ها \\
\hline - / 19AMT & . & ارتفاع \\
\hline F/IDTMTF & $F / r \cdot \Delta r q V$ & زمان به قله رسيدن \\
\hline T/RQITA & T/GOTKIA & عرض \\
\hline - $11 \cdot \Lambda \Delta T_{T}$ & $\cdot|| K \mid A V \cdot I$ & MSE \\
\hline$-r \cdot \Delta / q \cdot r \Delta$ & $-r \wedge q / \varepsilon \vee \Delta q$ & AIC \\
\hline$-r q V / \cdot V V q$ & $-r \Lambda \cdot|\Lambda \Delta| \cdot$. & SBC \\
\hline
\end{tabular}

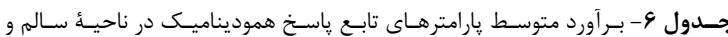

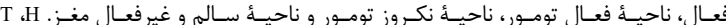

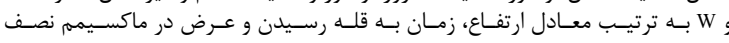

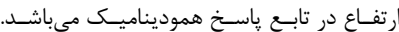

\begin{tabular}{|c|c|c|c|}
\hline مدل TD & مدل Lل & برآورد متوسط بار امترها & ناحيلبندى مغز \\
\hline$\cdot 1 \cdot 190$ & d. & $\mathrm{H}$ & \multirow{3}{*}{ ناحية سالم و فعال } \\
\hline $9(9 \cdot V)$ & $11 / 9 \cdot v 1$ & $\mathrm{~T}$ & \\
\hline$\Delta / \cdot r \Delta V$ & $\Delta / \Delta$ & W & \\
\hline$\cdot 11+\cdot v$ & $\cdot / \cdot \lambda r \cdot 1$ & $\mathrm{H}$ & \multirow{3}{*}{ ناحية فعال تومور } \\
\hline$\Delta / \cdot \Delta \Lambda \Lambda$ & $V / 9 V \cdot \Delta$ & $\mathrm{T}$ & \\
\hline f/IIVe & F & $\mathrm{W}$ & \\
\hline $.1 \cdot 191$ & $-\cdot / \cdot \Delta 11$ & $\mathrm{H}$ & \multirow{3}{*}{ ناحية نكروز تومور } \\
\hline$f / \cdot r \Delta V$ & W/IFYA & $\mathrm{T}$ & \\
\hline$r / r \Delta V /$ & 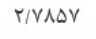 & W & \\
\hline .1 .199 & $\% \cdot 1 \Delta r$ & $\mathrm{H}$ & \multirow{3}{*}{ ناحية سالم و غير فعال } \\
\hline $9 / 0$ & $1 f / 9994$ & $\mathrm{~T}$ & \\
\hline $0 / 1999$ & r/9999 & $\mathrm{W}$ & \\
\hline
\end{tabular}




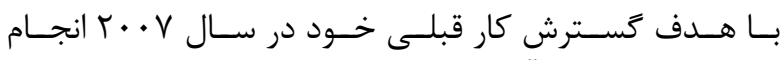

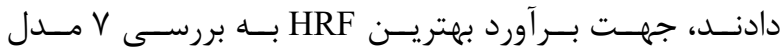

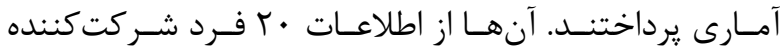

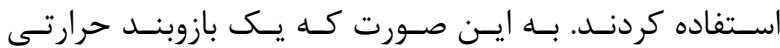

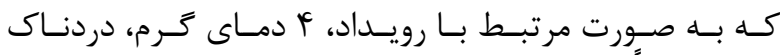

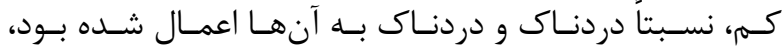

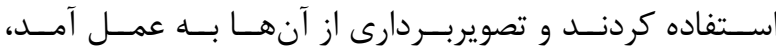

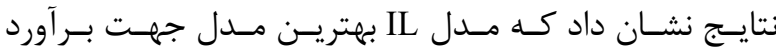

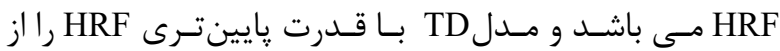

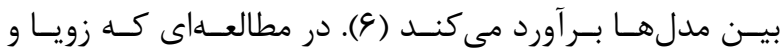

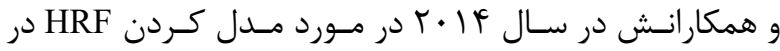

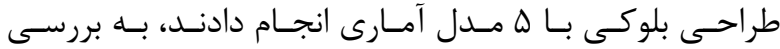

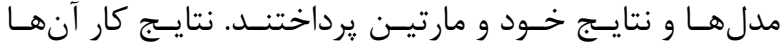

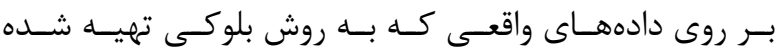

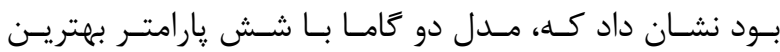

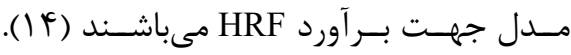

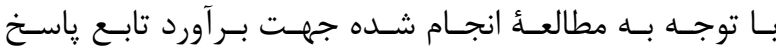

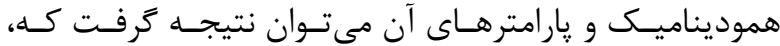

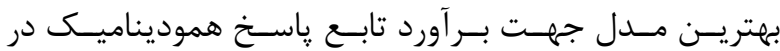

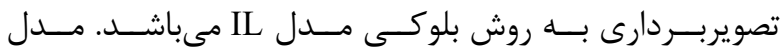

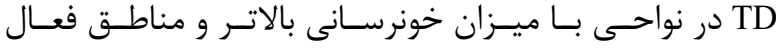

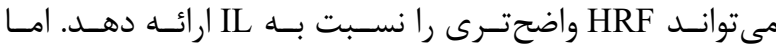

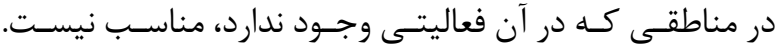

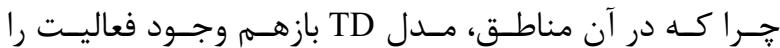

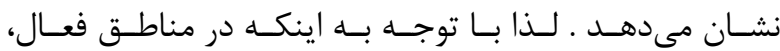

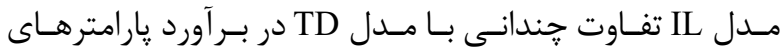

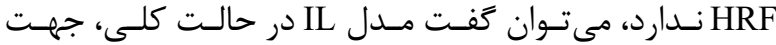

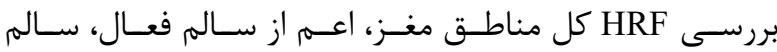

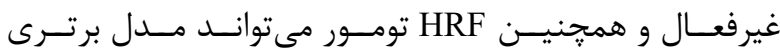

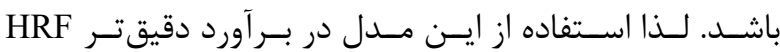

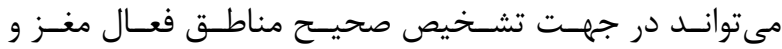

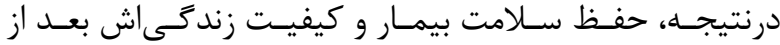

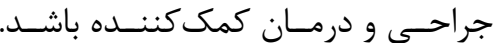

1. Aguirre GK, Zarahn E, D'esposito M. The variability of human, BOLD hemodynamic responses. Neuroimage. 1998; 8(4): 360-9.

2. Logothetis NK, Pauls J, Augath M, Trinath T, Oeltermann A. Neurophysiological investigation of the basis of the fMRI signal. Nature. 2001; 412(6843): 150-7.

3. Bellgowan PS, Saad ZS, Bandettini PA. Bandettini, Understanding neural system dynamics through task modulation and measurement of functional MRI amplitude, latency, and width. Proc Natl Acad Sci U S A. $2003 ; 100(3)$ : 1415-9.

4. Handwerker DA, Gonzalez-Castillo J, D'Esposito M, Bandettini PA. The continuing challenge of unders

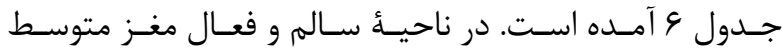

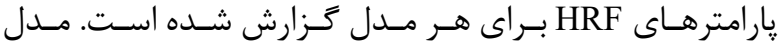

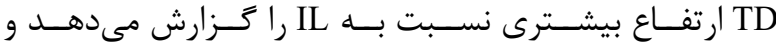

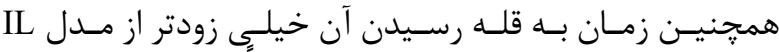

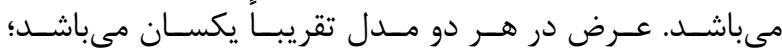

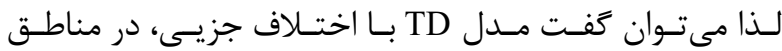

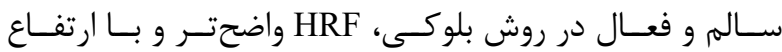

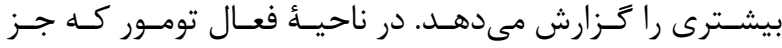

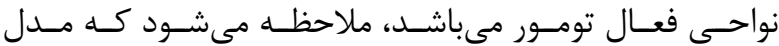

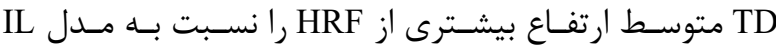

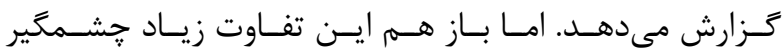

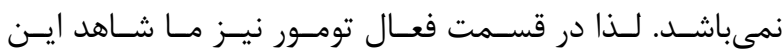

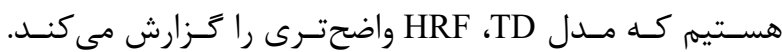

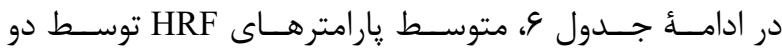

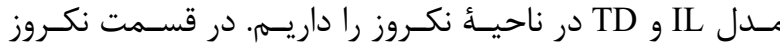

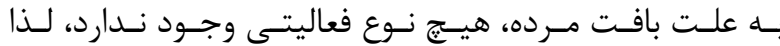

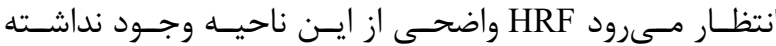

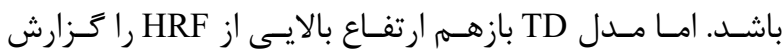

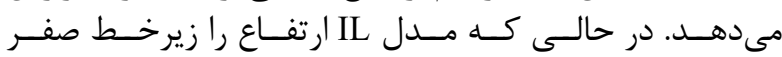

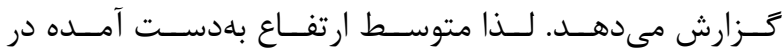

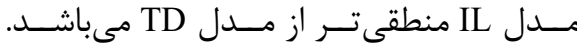

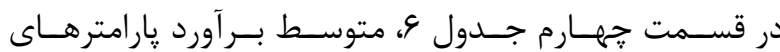

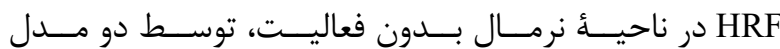

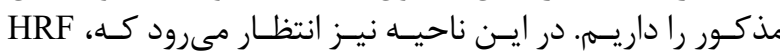

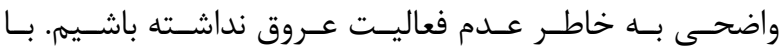

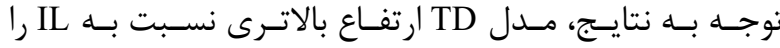

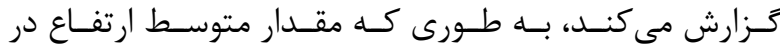

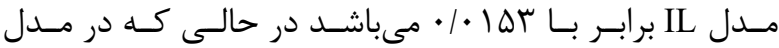

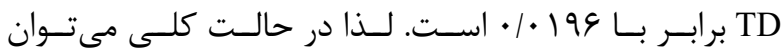

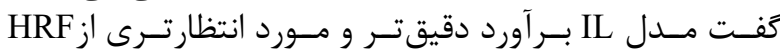

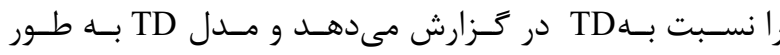

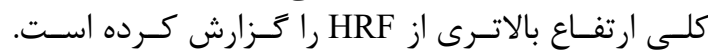

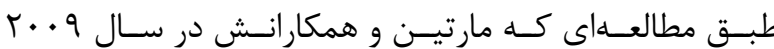

منابع

tanding and modeling hemodynamic variation in $\mathrm{fMRI}$. Neuroimage. 2012; 62(2): 1017-23.

5. Lindquist MA, Meng Loh J, Atlas LY, Wager TD. Modeling the hemodynamic response function in fMRI: efficiency, bias and mis-modeling. Neuroimage. 2009; 45(1): $187-98$

6. Muthukumaraswamy SD, Edden RAE, Jones DK, Swettenham JB, Singh KD. Resting GABA concentration predicts peak gamma frequency and fMRI amplitude in response to visual stimulation in humans. PNAS. 2009; 106(20): 8356-61.

7. D'Esposito M, Deouell LY, Gazzaley A. Alterations in the BOLD fMRI signal with ageing and disease: a 
challenge for neuroimaging. Nat Rev Neurosci. 2003; 4(11): 863-72.

8. Iadecola C. Neurovascular regulation in the normal brain and in Alzheimer's disease. Nat Rev Neurosci. 2004; 5(5): 347-60.

9. Maus B, van Breukelen GJ, Goebel R, Berger MP. Optimal design for nonlinear estimation of the hemodynamic response function. Hum Brain Mapp. 2012; 33(6): 1253-67.

10. Lindquist MA, Waugh C, Wager TD. Modeling state-related fMRI activity using change-point theory. NeuroImage. 2007; 35(3): 1125-41.
11. Friston KJ. Imaging neuroscience: Principles or maps? Proc Natl Acad Sci U S A. 1998; 95(3): 796-802.

12. Friston KJ, Glaser DE, Henson RNA, Kiebel S, Phillips C, Ashburner J. Classical and bayesian inference in neuroimaging: applications. Neuroimage. 2002; 16(2): 484-512.

13. Worsley KJ, Friston KJ. Friston, Analysis of fMRI time-series revisited-again. Neuroimage, 1995; 2(3): 173-81.

14. Shan ZY, Wright MJ, Thompson PM, McMahon KL, Blokland GG, de Zubicaray GI, et al. Modeling of the hemodynamic responses in block design fMRI studies. J Cereb Blood Flow Metab. 2014; 34(2): 316-24. 